\title{
Lymphatic Absorption of Docosahexaenoic Acid Given as Monoglyceride, Diglyceride, Triglyceride, and Ethyl Ester in Rats
}

\author{
Fumiaki BANNo $^{1, *}$, Shinji DoISAKI ${ }^{2}$, Nobutoshi SHIMIZU ${ }^{2}$ \\ and Kenshiro FuIMOTO ${ }^{1}$ \\ ${ }^{1}$ Graduate School of Agricultural Science, Tohoku University, \\ 1-1 Tsutsumidori-Amamiyamachi, Aoba, Sendai 981-8555, Japan \\ ${ }^{2}$ Central Research Laboratory, Nippon Suisan Kaisha, Ltd., \\ 559-6 Kitano-machi, Hachioji, Tokyo 192-0906, Japan
}

(Received March 9, 2001)

\begin{abstract}
Summary Lymphatic absorption of docosahexaenoic acids (DHA) given as monoglyceride (MG), consisting of 1 (or 3)-species (91.4\%), 2-species (4.2\%) and diglyceride (DG) consisting of 1,3 -species $(70.8 \%), 1$ (or 3),2-species $(28.6 \%)$, were investigated in comparison with that of triglyceride (TG) and ethyl ester (EE). Rats were infused with a lipid emulsion containing $200 \mathrm{mg}$ of DHA-MG, DG, TG, or EE via a gastric cannula. Lymph was collected through the thoracic lymph duct at $2 \mathrm{~h}$ intervals for $10 \mathrm{~h}$ and at a single collection from 10 to $30 \mathrm{~h}$. Physiological saline containing glucose was infused $(2 \mathrm{~mL} / \mathrm{h})$ throughout the lymph collection. The overall recovery of DHA at $30 \mathrm{~h}$ after its infusion was significantly higher in the rank order DHA-MG $>$ DG $>$ TG $=\mathrm{EE}$. Moreover, time-dependent changes in recovery rates from 2 to $10 \mathrm{~h}$ of DHA given as MG were significantly higher than those of the corresponding DG, TG, and EE. These results indicate that DHA-MG and DG are absorbed and transported more effectively than TG and EE forms under restricted water supply, even if they mainly consist of 1 (or 3)-species. Lymph lipids were mainly transported as TG, and a large amount of DHA was incorporated into a TG fraction in all fat types examined. Furthermore, the intramolecular distribution of DHA in lymph TG was similar in all groups. Key Words docosahexaenoic acid, monoglyceride, diglyceride triglyceride, ethyl ester
\end{abstract}

Long-chain $n-3$ highly unsaturated fatty acids (HUFA), such as eicosapentaenoic (EPA) and docosahexaenoic (DHA) acids, are known to have several important physiological and pharmacological functions $(1,2)$. In vitro studies have demonstrated that $n-3$ HUFA may exhibit resistance toward hydrolysis in the gastrointestinal tract $(3,4)$, and it has thus been speculated that DHA in triglyceride (TG) may not be efficiently digested and absorbed. Most dietary TGs are digested into fatty acids and 2-monoglyceride (MG) mainly by the $s n-1,3$ specific pancreatic lipase. They are absorbed into intestinal mucosal cells and resynthesized to TGs, which are assembled into chylomicrons and secreted into lymph. Recent studies showed that the intestinal absorption of DHA and EPA given as ethyl ester (EE) was lower than seen in the case of TG or free acid $(5,6)$. It was also reported that the absorption of DHA and EPA at the $s n-1,3$ positions of TG was lower than when these acids were esterified at the $s n-2$ position of TG (7-9). These results suggest that in the case of a single injection, the absorption of DHA might be limited by lipase hydrolysis in vivo. In infants (10) and patients with pancreatic dysfunction (11), a disorder of digestion and the absorption of HUFA-containing TG was suggested because of the insufficient secretion of pancreatic lipase. From this point of view, we thought that

* To whom correspondence should be addressed. the MG or diglyceride (DG) type of lipid molecules containing DHA might be favorable in terms of absorption and utilization efficiency. The purpose of this study was to compare the absorption of DHA given as MG, DG, TG, and $\mathrm{EE}$ forms by the direct measurement of DHA in the lymph of the thoracic duct cannulated rats with the restriction of water supply to $2 \mathrm{~mL} / \mathrm{h}$. Because under this condition the secretion of lymph is insufficient and the absorption of DHA is retarded, the effects of the glyceride structure on the absorption of DHA will be clearly demonstrated.

\section{MATERIALS AND METHODS}

Preparation of administered lipids. DHA-EE (purity $>70 \%)$ was prepared by the Central Research Laboratory, Nippon Suisan Kaisha, Ltd., Hachioji, Tokyo, Japan. Free DHA was prepared from DHA-EE by saponification. DHA-MG, DG, and TG were chemically synthesized by the esterification of free DHA with glycerol in the presence of 4-dimethylaminopyridine (DMAP) and $N, N^{\prime}$-dicyclohexylcarbodiimide (DCC) as catalysts (12). The reaction was carried out at room temperature in an atmosphere of nitrogen, and the molar ratio of DHA, glycerol, DMAP, and DCC was 1/4/4/1 for MG, $1 / 1 / 1.5 / 1$ for DG, and 3/1/1/3 for TG. Synthesized glycerides were purified through a silica gel column and stored at $-30^{\circ} \mathrm{C}$ before gastric injection.

Animals and lymph collection. Male Sprague-Dawley 
rats (CLEA Japan Inc., Tokyo, Japan) weighing 300$370 \mathrm{~g}$ were fed a commercial nonpurified diet (F-2; Funabashi Farms, Chiba, Japan) and given drinking water ad libitum until the day of operation. The rats were anesthetized with pentobarbital $(35 \mathrm{mg} / \mathrm{kg}$ body weight, i.p.), and the left thoracic lymph duct was cannulated according to the procedure of Ballman et al. (13). Another catheter was inserted into the fundus of the stomach. After surgery, the rats were placed in restraining cages in an air-conditioned room $\left(20-25^{\circ} \mathrm{C}\right)$. A normal osmotic solution containing $139 \mathrm{~mm}$ glucose and $85 \mathrm{~mm} \mathrm{NaCl}$ was continuously infused through the gastric cannula $(2 \mathrm{~mL} / \mathrm{h})$ until the end of this experiment (5). The rats had no access to drinking water to get constant lymph flow and to retard the absorption of TG and other lipids. The next morning, the collection of lymph for $2 \mathrm{~h}$ provided a baseline, and the rats were infused with $2 \mathrm{~mL}$ of a test emulsion via the gastric cannula. The test emulsion contained $200 \mathrm{mg}$ of sodium cholate, and $200 \mathrm{mg}$ of DHA-MG, DG, TG, or EE was prepared by sonication. After an administration of the emulsion, lymph was collected in a tube (which was kept on ice) containing EDTA at $2 \mathrm{~h}$ intervals for $10 \mathrm{~h}$ and at a single collection from 10 to $30 \mathrm{~h}$. The lymph samples were then stored at $-30^{\circ} \mathrm{C}$ until analyzed.

Lipid analyses. Total lipids were extracted from the aliquots of lymph according to the procedure of Folch et al. (14) in the presence of butylated hydroxytoluene $(0.01 \%)$ as an antioxidant. Fractions of cholesterol ester (Chl-E), TG, and phospholipid (PL) were separated by thin-layer chromatography (TLC), using hexane/diethyl ether/acetic acid $(80: 20: 1, \mathrm{v} / \mathrm{v} / \mathrm{v})$ as a developing solvent. Fatty acids were methylated in methanol/benzene $(4: 1, \mathrm{v} / \mathrm{v})$ in the presence of acetyl chloride with heptadecanoic acid $(17: 0)$ as the internal standard (15). Fatty acid methyl esters were analyzed by gas-liquid chromatography (GLC), using a GC-380 chromatograph (GL Sciences, Tokyo, Japan) equipped with a fused silica capillary column (CP-SIL88; $0.25 \mathrm{~mm}$ i.d. $\times 50 \mathrm{~m}$, Chrompack, Middleburg, Netherlands). A hydrogen flame ionization detector (FID) was used with an injection temperature of $250^{\circ} \mathrm{C}$, and the column temperature was programmed from 170 to $225^{\circ} \mathrm{C}$ at $4^{\circ} \mathrm{C} / \mathrm{min}$. The DHA recovery rate was calculated by subtracting a DHA amount in the baseline lymph from that in the lymph collected after the administration of the test emulsion.

The positional distribution of fatty acids in lymph TG was determined by the Grignard degradation method $(9,16)$. TGs $(5-10 \mathrm{mg})$ were dissolved in $0.6 \mathrm{~mL}$ of anhydrous diethyl ether, and $60 \mu \mathrm{L}$ of $3 \mathrm{M}$ ethylmagnesium bromide in diethyl ether was added. The mixture was shaken for $30 \mathrm{~s}$, and $0.25 \mathrm{~mL}$ of acetic acid/diethyl ether $(1: 1, \mathrm{v} / \mathrm{v})$ was then added to stop the reaction. The products were extracted by diethyl ether saturated with boric acid. The ether extract was washed twice with $2 \%$ aqueous sodium bicarbonate, then washed with water and dried over anhydrous sodium sulfate. After removal of the solvent under nitrogen, 2-MGs were isolated by TLC on $5 \%$ boric-acid-impregnated sil-
Table 1. Lipid composition (wt\%) of administered lipids.

DHA-MG DHA-DG DHA-TG DHA-EE

\begin{tabular}{lcccc} 
1(3)-MG & 91.4 & - & - & - \\
2-MG & 4.2 & - & - & - \\
1,2(2,3)-DG & - & 28.6 & - & - \\
1,3-DG & - & 70.8 & - & - \\
TG & - & - & 99.1 & - \\
EE & - & - & - & 100 \\
\hline
\end{tabular}

Table 2. Fatty acid composition (mol\%) of administered lipids.

\begin{tabular}{lrrrr}
\hline & DHA-MG & DHA-DG & DHA-TG & DHA-EE \\
\hline $22: 1$ & 2.1 & 2.2 & 2.5 & 5.1 \\
$22: 5(n-3)$ & 20.6 & 19.3 & 18.8 & 17.3 \\
$22: 6(n-3)$ & 74.3 & 75.1 & 74.9 & 71.9 \\
\hline
\end{tabular}

ica gel plates with chloroform/acetone $(88: 12, \mathrm{v} / \mathrm{v})$ as a developing solvent. The 2-MG fraction was transmethylated, and the fatty acid methyl ester was analyzed by GLC, as described above.

Major lipid classes in lymph lipids and administered lipids were measured by a TLC-FID system using an Iatroscan TH-10 (Iatron, Tokyo, Japan) with silica gel rods S-III (Iatron). A mixture of hexane/diethyl ether/ acetic acid $(80: 20: 1, \mathrm{v} / \mathrm{v} / \mathrm{v})$ was used as a developing solvent; however, isomeric MGs were separated by $3 \%$ boric-acid-impregnated silica gel rods with chloroform/acetone $(96: 4, \mathrm{v} / \mathrm{v})$ as a mobile phase (17).

Statistical analysis. The results were expressed as mean $\pm S E$, and the differences were assessed by oneway analysis of variance followed by Duncan's new multiple-range test.

\section{RESULTS}

The lipid composition and fatty acid composition of administered lipids are shown in Table 1 and Table 2. The concentration of $s n-1(3)-M G$ in DHA-MG was higher than $90 \%$, and the concentration of $s n-1,3-\mathrm{DG}$ in DHA-DG was higher than 70\%. The purities of DHATG and EE were almost $100 \%$. The DHA concentrations of all preparations were approximately $70 \%$, and a major impurity was docosapentaenoic acid (about $20 \%)$.

Lymph flow rates were similar for each group (mean values $1.9 \pm 0.4 \mathrm{~mL} / \mathrm{h}, 2.0 \pm 0.3 \mathrm{~mL} / \mathrm{h}, 1.7 \pm 0.1 \mathrm{~mL} / \mathrm{h}$, and $1.7 \pm 0.2 \mathrm{~mL} / \mathrm{h}$ for DHA-MG, DG, TG, and EE groups, respectively). The lymphatic recovery rates of DHA were calculated from the infused dose and transported DHA (Fig. 1). Overall recovery at $30 \mathrm{~h}$ after infusion was significantly higher in the following order: DHA-MG $>$ DG $>$ TG $=$ EE. Moreover, time-dependent changes in the recovery rates $(2-10 \mathrm{~h})$ of DHA given as MG were significantly higher than those of the corresponding DG, TG, and EE. Because the overall recovery of DHA in any position of TG was reported to be very 


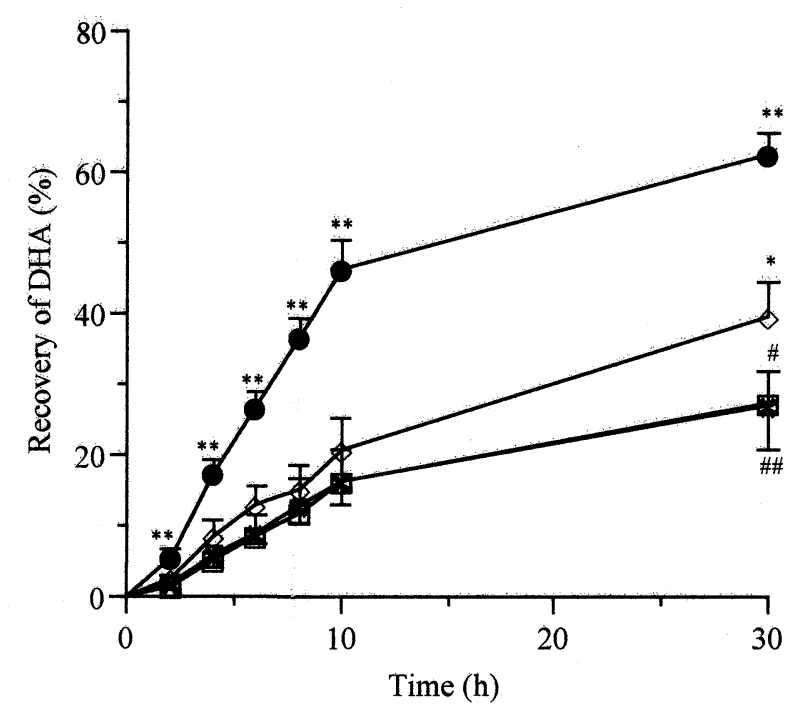

Fig. 1. Lymphatic recovery of DHA in the thoracic duct lymph of rats after intragastric administration of a different lipid emulsion. 0 , DHA-MG $(n=4) ; \diamond$, DHA-DG $(n=5) ; \square$, DHA-TG $(n=6) ; \mathbf{X}$, DHA-EE $(n=4)$. Data are mean \pm SE. * Significantly higher than DHA-TG and EE at $p<0.05$. ${ }^{* *}$ Significantly higher than DHA-DG, TG, and EE at $p<0.05$. " SE of DHA-TG,

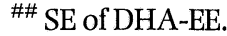

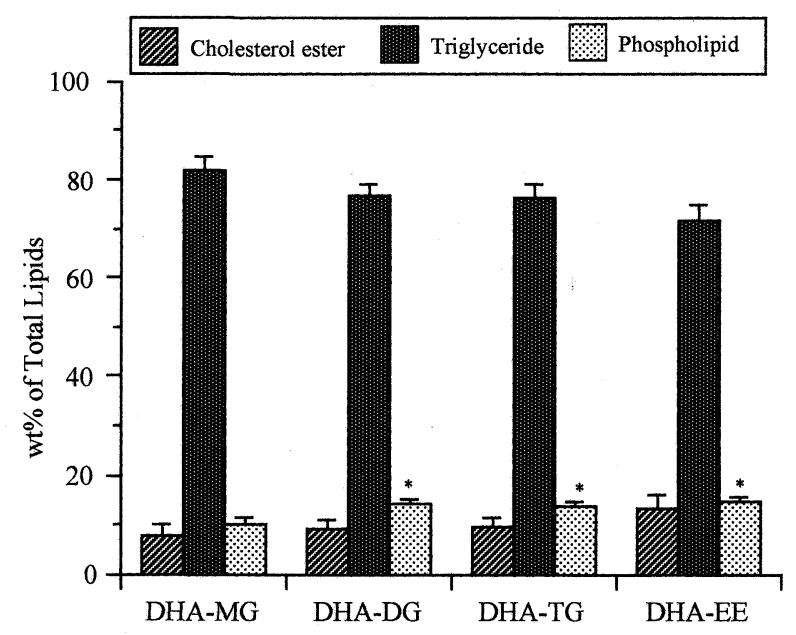

Fig. 2. Lipid composition (wt\%) of lymph lipids in rats after intragastric administration of a different lipid emulsion. DHA-MG $(n=4)$; DHA-DG $(n=5)$; DHA-TG $(n=5) ; \quad$ DHA-EE $(n=4)$. Data are mean \pm SE. * Significantly different from DHA-MG at $p<0.05$.

well under the sufficient water supply $(6,7)$, the recovery of DHA of TG in the present study was poor as a result of the restricted water supply.

The lipid composition of lymph lipids collected during $2-10 \mathrm{~h}$ after the infusion of test emulsions is shown in Fig. 2. The lymph lipids were mainly transported as TG regardless of the lipid type. MG and DG were not detectable or traced in lymph, even after these lipids had been administered. The proportion of lymph lipids transported as PL was significantly higher in the DHADG, TG, and EE groups than in the DHA-MG group. The ratio of Chl-E fractions in the total lipids was not signifi-

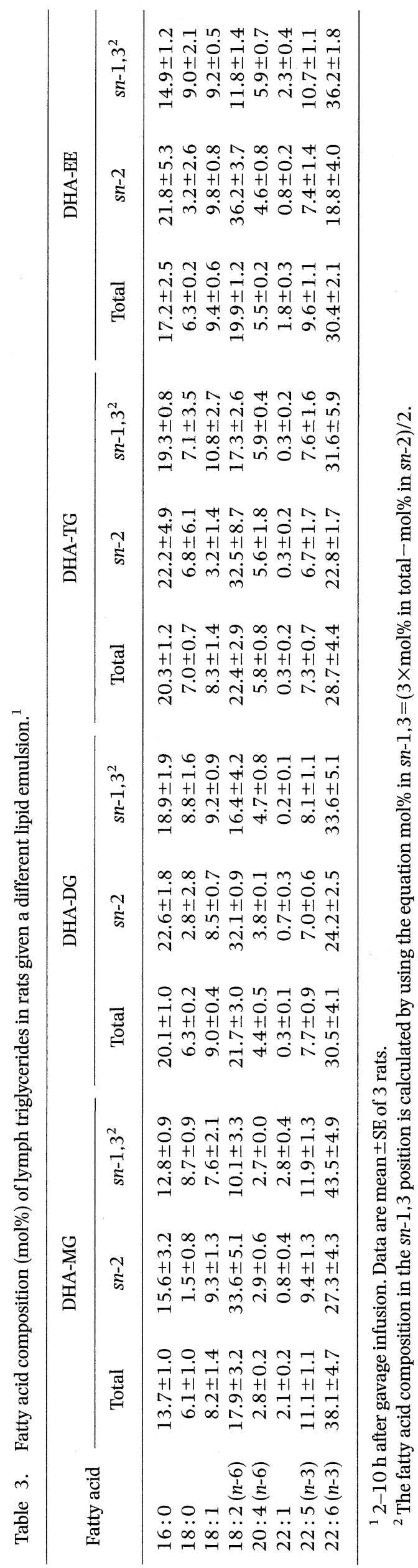


Table 4. Fatty acid composition (mol\%) of lymph phospholipids in rats given a different lipid emulsion. ${ }^{1}$

\begin{tabular}{lrrrr}
\hline Fatty acid & DHA-MG & DHA-DG & DHA-TG & DHA-EE \\
\hline $16: 0$ & $22.7 \pm 0.9$ & $22.9 \pm 1.2$ & $24.0 \pm 0.4$ & $25.0 \pm 0.7$ \\
$18: 0$ & $17.4 \pm 0.5$ & $18.7 \pm 0.6$ & $19.6 \pm 0.4$ & $18.0 \pm 0.4$ \\
$18: 1$ & $6.8 \pm 0.7$ & $5.4 \pm 0.3$ & $4.8 \pm 0.1$ & $6.1 \pm 0.5$ \\
$18: 2(n-6)$ & $26.3 \pm 3.7$ & $22.6 \pm 1.0$ & $21.8 \pm 2.4$ & $21.1 \pm 1.3$ \\
$20: 4(n-6)$ & $14.0 \pm 2.2$ & $19.6 \pm 1.2$ & $20.8 \pm 0.4$ & $20.6 \pm 1.0$ \\
$22: 1$ & tr & $\operatorname{tr}$ & $\operatorname{tr}$ & $\operatorname{tr}$ \\
$22: 5(n-3)$ & $2.4 \pm 0.3$ & $1.4 \pm 0.2$ & $1.0 \pm 0.1$ & $1.7 \pm 0.3$ \\
$22: 6(n-3)$ & $10.5 \pm 1.6$ & $9.4 \pm 1.2$ & $8.0 \pm 1.3$ & $7.6 \pm 0.9$ \\
\hline
\end{tabular}

${ }^{1} 2-10 \mathrm{~h}$ after gavage infusion. Data are mean \pm SE of 3 rats. tr, trace.

Table 5. Fatty acid composition (mol\%) of lymph cholesterol esters in rats given a different lipid emulsion. ${ }^{1}$

\begin{tabular}{lcrcc}
\hline Fatty acid & DHA-MG & DHA-DG & DHA-TG & DHA-EE \\
\hline $16: 0$ & $27.9 \pm 1.8$ & $27.5 \pm 2.5$ & $21.3 \pm 3.7$ & $31.3 \pm 2.2$ \\
$18: 0$ & $10.1 \pm 0.7$ & $9.1 \pm 0.9$ & $18.7 \pm 10.4$ & $10.9 \pm 1.2$ \\
$18: 1$ & $21.5 \pm 1.6$ & $21.6 \pm 0.5$ & $16.9 \pm 4.2$ & $22.4 \pm 1.6$ \\
$18: 2(n-6)$ & $27.8 \pm 3.2$ & $29.6 \pm 2.4$ & $26.9 \pm 5.7$ & $25.0 \pm 1.4$ \\
$20: 4(n-6)$ & $9.0 \pm 3.4$ & $10.6 \pm 1.5$ & $13.5 \pm 3.9$ & $9.7 \pm 3.1$ \\
$22: 1$ & $\operatorname{tr}$ & $\operatorname{tr}$ & $\operatorname{tr}$ & $\operatorname{tr}$ \\
$22: 5(n-3)$ & $\operatorname{tr}$ & $\operatorname{tr}$ & $\operatorname{tr}$ & $\operatorname{tr}$ \\
$22: 6(n-3)$ & $3.6 \pm 1.0$ & $1.6 \pm 0.1$ & $2.7 \pm 1.0$ & $0.8 \pm 0.1$ \\
& & & & \\
\hline
\end{tabular}

${ }^{1} 2-10 \mathrm{~h}$ after gavage infusion. Data are mean \pm SE of 3 rats. tr, trace.

cantly different among the four DHA groups.

Fatty acid compositions of major lipid classes in lymph lipids collected during $2-10 \mathrm{~h}$ after an infusion of the test emulsions are shown in Tables 3-5. The DHA content by mol\% tended to be highest in the TG fraction and lowest in the Chl-E fraction, irrespective of administered lipid molecules. In all lipid classes, palmitic acid, stearic acid, oleic acid, linoleic acid, and aracidonic acid were the major endogenous fatty acids that were not included in administered lipids; in particular, stearic acid, oleic acid, and aracidonic acid concentrations differed with lipid classes.

When DHA was given in any form, DHA (57-65\%) tended to be at the $s n-1,3$ position of TG in lymph that was collected $2-10 \mathrm{~h}$ after infusion (Fig. 3). In contrast, linoleic acid was mainly esterified in the $s n-2$ position regardless of the injection form (Table 3).

\section{DISCUSSION}

In the present study, rats were administered the emulsion containing DHA-MG or DG mainly composed of sn-1,3-species under the restriction of water supply. These lipid molecules are considered to be cleaved to fatty acids and glycerols by the $s n-1,3$ specific lipase, and no 2-MG will be formed. Therefore the absorbed fatty acids are suggested to be reconstituted to TG exclusively through the glycerol-3-phosphate pathway. On the other hand, most fatty acids in the dietary TG

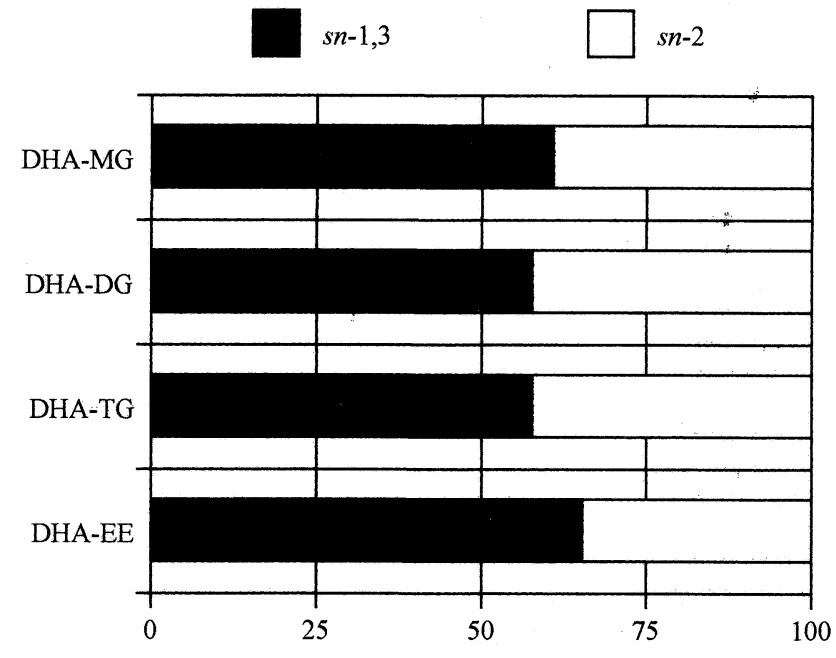

Fig. 3. Intramolecular distribution of DHA in rat lymph triglyceride. Data are mean of 3 rats.

are absorbed and reconstituted to TG via the 2-monoglyceride pathway. Since the 2-monoglyceride pathway is more active than the glycerol-3-phosphate pathway in the intestinal mucosal cells (18), it is possible that sn1(3)-MG and sn-1,3-DG would rather be less absorbable than the TG form. Murata et al. (19) have shown that an intragastric infusion of an emulsion containing DG mainly consisting of 1,3-species, which had been synthesized by fatty acids from rapeseed oil, significantly retarded the lymphatic transport of TG and cholesterol in rats compared to that containing rapeseed oil TG. But in the present study, when DHA was given as a sole fatty acid, the lymphatic recoveries of DHA given in the form of MG and DG were significantly higher than that of TG and EE. Especially, the overall recovery rates of DHA given as MG were about twofold higher than those of the corresponding TG and EE. In rapeseed oil, oleic acid (about 60\%) and linoleic acid (about 20\%) were the chief constituent fatty acids. They are quickly digested and absorbed almost quantitatively under normal conditions. In this case, because the lack of 2monoglyceride on resynthesis of triglyceride may be rate limiting, the absorption rate of sn-1,3-DG would be lower than that of TG. But it has been reported that the activity of pancreatic lipase on esters of long-chain HUFAs, such as DHA and EPA, is less prominent, and the action of carboxylesterase may be more important in the hydrolysis of glycerides comprised of these fatty acids $(20,21)$. Thus in DHA-containing lipid molecules, the relative importance of enzymes involved in digestion processes may be different from other common fatty acids, and DHA-TG (tridocosahexaenoin) is then not efficiently digested. For this reason, DHA-MG and DG were absorbed more effectively than the TG and EE forms, even if most fatty acids of MG or DG are distributed at the $s n-1$ or $s n-3$ position, as in this study. On the other hand, a possible reason for the poor recovery of DHA given as TG or EE was the restriction of water. In the present study, water is supplied only through a stomach tube at $2 \mathrm{~mL} / \mathrm{h}$. Since the lymph flow was in- 
sufficient under this condition, the difference in the absorbability of lipids was suggested to be emphasized in comparison with the normal condition.

When DHA was given as $s n-1$ (3)-MG, $s n-1,3-\mathrm{DG}$, or $\mathrm{EE}$, most TG containing DHA in chylomicron would be synthesized in intestinal mucosal cells via the glycerol3-phosphate pathway. Because this pathway is also the predominant pathway for synthesizing PL in chylomicron (22), it could be expected that DHA in these forms, compared with the TG form, was efficiently incorporated into PL fractions in lymph lipids. However, a large portion of DHA administered was distributed in the TG fraction, regardless of the structure of administered lipid molecules. Thus the structural differences in the DHAcontaining lipids were not reflected in the distribution of DHA in the lipids of lymph chylomicrons in this experiment.

During the absorption process of dietary TG, acyl chains are not completely hydrolyzed to free fatty acids, but the greater part (about $80 \%$ ) of the fatty acids in the $s n-2$ position are conserved in the original position (23). In our study, the conservation of the $s n-2$ position of DHA during absorption was almost $85 \%$, following the administration of DHA-TG. Therefore our observation was consistent with previous studies in this respect. Yang and Kuksis reported that DHA was mainly distributed at the $s n-1$ and 3 positions in chylomicron TG from rats given menhaden oil fatty acid methyl or ethyl esters, which would be expected to be biosynthesized via the glycerol-3-phosphate pathway (24). However, when DHA was given as MG, DG, and EE forms in this study, a large amount of DHA was incorporated into the $s n-2$ position of lymph TG. This discrepancy is attributable to the differences in the fatty acid composition of administered lipids. The menhaden oil contains a high amount of DHA (8-9 mol\%), but there are also large amounts of other unsaturated fatty acids, such as palmitoleic $(16-18 \mathrm{~mol} \%)$, oleic $(9-10 \mathrm{~mol} \%)$, and eicosapentaenoic (15-17 mol\%) acids. Ikeda et al. suggested that DHA was incorporated almost exclusively at the sn-1 and 3 positions in lymph TG when oleic acid or triolein was simultaneously given with ethyl DHA (25). Therefore under the coexistence of other unsaturated fatty acids, DHA is specifically bound to the $s n-1$ and 3 positions in TG synthesis via the glycerol-3-phosphate pathway. On the other hand, Ikeda et al. also provided the evidence that a small portion $(\sim 27 \%)$ of DHA was incorporated into the $s n-2$ position of TG in lymph when ethyl DHA was given as a sole lipid (25). This result is consistent with our findings in which about $35 \%$ of DHA was distributed in the $s n-2$ position in the lymph TG of rats given DHA-EE. Thus there is the possibility that when other dietary fatty acids bound to the $s n-2$ position of TG are insufficient, part of the DHA might be incorporated into the sn-2 position via the glycerol-3-phosphate pathway. Since little information is available on fatty acid specificity, especially on DHA in TG resynthesis via the glycerol-3-phosphate pathway in intestinal mucosal cells, more detailed studies are necessary to confirm this hypothesis.
In conclusion, these results indicate that DHA-MG and DG are absorbed and transported more effectively than DHA-TG and EE under a water-restricted condition. Further studies on the effect of long-term consumption and of coexisting fats on the recovery of DHA are needed to confirm our conclusion.

\section{REFERENCES}

1) Herold PM, Kinsella JE. 1986. Fish oil consumption and decreased risk of cardiovascular disease: a comparison of findings from animal and human feeding trial. $\mathrm{Am} \mathrm{J}$ Clin Nutr 43: 566-598.

2) Simopoulos AP. 1991. Omega-3 fatty acids in health and disease and in growth and development. Am J Clin Nutr 54: 438-463.

3) Brockerhoff H, Hoyle RJ, Huang PC. 1966. Positional distribution of fatty acids in the fats of a polar bear and seal. Can J Biochem 44: 1519-1525.

4) Bottino NR, Vandenburg GA, Reiser R. 1967. Resistance of certain long chain polyunsaturated fatty acids of marine oils to pancreatic lipase hydrolysis. Lipids 2: 489-493.

5) Ikeda I, Imasato Y, Inayoshi A, Nagao H, Sasaki E, Sugano M, Imaizumi K, Yazawa K. 1993. Lymphatic transport of eicosapentaenoic and docosahexaenoic acids as triglyceride, ethyl ester and free acid, and their effect on cholesterol transport in rats. Life Sci 52: 1371-1379.

6) Ikeda I, Sasaki E, Yasunami H, Nomiyama S, Nakayama M, Sugano M, Imaizumi K, Yazawa K. 1995. Digestion and lymphatic transport of eicosapentaenoic and docosahexaenoic acids given in the form of triacylglycerol, free acid and ethyl ester in rats. Biochim Biophys Acta 1259: $297-304$

7) Christensen MS, Høy C-E, Redgrave TG. 1994. Lymphatic absorption of $n-3$ polyunsaturated fatty acids from marine oils with different intramolecular fatty acid distributions. Biochim Biophys Acta 1215: 198-204.

8) Christensen MS, Høy C-E, Becker CC, Redgrave TG. 1995. Intestinal absorption and lymphatic transport of eicosapentaenoic (EPA), docosahexaenoic (DHA), and decanoic acids: dependence on intramolecular triacylglycerol structure. Am J Clin Nutr 61: 56-61.

9) Yoshida H, Kumamaru J, Mawatari M, Ikeda I, Imaizumi K, Tsuji H, Seto A. 1996. Lymphatic absorption of seal and fish oils and their effect on lipid metabolism and eicosanoid production in rats. Biosci Biotech Biochem 60: 1293-1298.

10) Boehm G, Müller H, Kohn G, Moro G, Minoli I, Böhles HJ. 1997. Docosahexaenoic and arachidonic acid absorption in preterm infants fed LCP-free or LCP-supplemented formula in comparison to infants fed fortified breast milk. Anal Nutr Metab 41: 235-241.

11) Christensen MS, Mullertz A, Høy C-E. 1995. Absorption of triglycerides with defined or random structure by rats with biliary and pancreatic diversion. Lipids 30: 521-526.

12) Awl RA, Frankel EN, Weisleder D. 1989. Synthesis and characterization of triacylglycerols containing linoleate and linolenate. Lipids 24: 866-872.

13) Bollman JL, Cain JC, Grindlay JH. 1948. Techniques for the collection of lymph from the liver, small intestine, or thoracic duct of the rat. J Lab Clin Med 33: 1349-1352.

14) Folch J, Lees M, Sloane-Stanley GH. 1957. A simple 
method for the isolation and purification of total lipids from animal tissues. J Biol Chem 226: 497-509.

15) Lepage G, Roy CC. 1986. Direct transesterification of all classes of lipids in a one-step reaction. J Lipid Res 27: 114-120.

16) Myher JJ, Kuksis A. 1979. Stereospecific analysis of triacylglycerols via racemic phosphatidylcholines and phospholipase C. Can J Biochem 57: 117-124.

17) Tanaka M, Itoh T, Kaneko H. 1980. Quantitative determination of isomeric glycerides, free fatty acids and triglycerides by thin layer chromatography-flame ionization detector system. Lipids 15: 872-875.

18) Tso P. 1985. Gastrointestinal digestion and absorption of lipid. Adv Lipid Res 21: 143-185.

19) Murata M, Hara K, Ide T. 1994. Alteration by diacylglycerols of the transport and fatty acid composition of lymph chylomicrons in rats. Biosci Biotech Biochem 58: 1416-1419.

20) Chen Q, Sternby B, Nilsson A. 1989. Hydrolysis of triacylglycerol arachidonic and linoleic acid ester bonds by human pancreatic lipase and carboxyl ester lipase.
Biochim Biophys Acta 1004: 372-385.

21) Chen Q, Sternby B, Akesson B, Nilsson A. 1990. Effects of human pancreatic lipase-colipase and carboxyl ester lipase on eicosapentaenoic and arachidonic acid ester bonds of triacylglycerols rich in fish oil fatty acids. Biochim Biophys Acta 1044: 111-117.

22) Small DM. 1991. The effects of glyceride structure on absorption and metabolism. Annu Rev Nutr 11: 413-434.

23) Kodali DR, Tercyak A, Fahey DA, Small DM. 1990. Acyl migration in 1,2-dipalmitoyl-sn-glycerol. Chem Phys Lipids 52: 163-170.

24) Yang LY, Kuksis A. 1991. Apparent convergence (at 2monoacylglycerol level) of phosphatidic acid and 2monoacylglycerol pathways of synthesis of chylomicron triacylglycerols. J Lipid Res 32: 1173-1186.

25) Ikeda I, Yoshida H, Imaizumi K. 1997. Effect of triolein or oleic acid on lymphatic recovery of docosahexaenoic acid given as ethyl ester and their intramolecular distribution in lymph triglyceride of rats. Lipids 32: 949952. 